ANDRADE, G.M., MOURA, M.S. e BARBOSA, F.C. Eficácia do produto homeopático Verm $100 \AA$ no controle da verminose ovina: resultados parciais. PUBVET, Londrina, V. 5, N. 8, Ed. 155, Art. 1046, 2011.

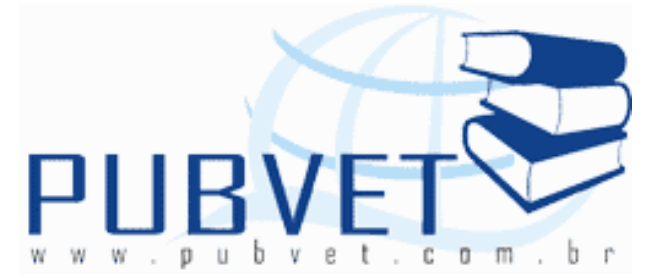

PUBVET, Publicações em Medicina Veterinária e Zootecnia.

\title{
Eficácia do produto homeopático Verm $100 \otimes$ no controle da verminose ovina: resultados parciais
}

Gabriella Mamede Andrade $^{1 *}$, Mariela Silva Moura ${ }^{1}$, Fernando Cristino Barbosa ${ }^{2}$

${ }^{1}$ Graduanda em Medicina Veterinária, Universidade Federal de Uberlândia. Uberlândia, MG.

${ }^{2}$ Docente da Faculdade de Medicina Veterinária, Universidade Federal de Uberlândia. Uberlândia, MG.

*Autor para correspondência: Rua Ceará, s/n, Bloco 2D, CEP 38400-902, Uberlândia, MG, Brasil. E-mail: gabriellamamede@gmail.com

\section{Resumo}

O controle de parasitas gastrintestinais na ovinocultura assim como em outros sistemas de criação representa quedas significativas na produtividade quando não é feito de forma adequada. Devido à crescente resistência dos parasitas aos anti-helmínticos comerciais alternativas como a utilização de medicamentos homeopáticos tem se destacado nos sistemas de produção. Este estudo teve como objetivo testar a eficácia do medicamento homeopático, VERM $100{ }^{\circledR}$, no controle dos parasitas gastrintestinais de 50 ovinos, em uma fazenda no município de Prata, Minas Gerais. Os dados foram obtidos através da contagem mensal de ovos por gramas de fezes (OPG), de animais distribuídos em dois grupos, sendo um grupo de animais controle e o outro de animais em tratamento. Os resultados foram interpretados estatisticamente 
ANDRADE, G.M., MOURA, M.S. e BARBOSA, F.C. Eficácia do produto homeopático Verm $100 \AA$ no controle da verminose ovina: resultados parciais. PUBVET, Londrina, V. 5, N. 8, Ed. 155, Art. 1046, 2011.

por meio de análise de variância (teste F) de um e 5\% de probabilidade e de regressão linear. As médias dos fatores qualitativos foram comparadas pelo teste de Tukey em nível de um e $5 \%$ de probabilidade. Não houve diferença estatisticamente significativa $(p>0,05)$ entre as médias de OPG dos dois grupos, tratado e controle. Contudo, o medicamento VERM $100 \AA$, do laboratório Real $\mathrm{H}$, não reduziu o número de ovos de nematódeos gastrintestinais, porém os ovinos não desenvolveram sintomas da parasitose.

Palavras-chave: Homeopatia, manejo, ovinos, parasitas gastrintestinais

\title{
Efficacy of homeopathic product Verm $100 \otimes$ in the control of sheep worms: partial results
}

\begin{abstract}
The control of gastrointestinal parasites in the sheep industry as well as other farming systems represents significant decrease in productivity when it is not done properly. Due to the increasing parasite resistance to anthelmintics commercial alternatives such as the use of homeopathic medicines are highlighted in production systems. This study aimed to test the efficacy of homeopathic medicine, VERM $100 \AA$, control of gastrointestinal parasites of sheep on a farm in the municipality of Prata, Minas Gerais. Data were collected through the monthly count of eggs per gram of feces (OPG), animals divided into two groups, one group of control animals and other animals undergoing treatment. The results were interpreted by analysis of variance ( $F$ test) and a $5 \%$ probability and linear regression. The means of qualitative factors were compared by Tukey test level and a $5 \%$ probability. There was no statistically significant difference ( $p>0.05)$ between the mean OPG of the two groups, treated and control. However, the drug VERM $100 \AA$, Real H Lab did not reduce the number of eggs of gastrointestinal nematodes, but the sheep did not develop symptoms of infection.
\end{abstract}

Keywords: Homeopathy, management, sheep, gastrointestinal parasites 
ANDRADE, G.M., MOURA, M.S. e BARBOSA, F.C. Eficácia do produto homeopático Verm $100 \AA$ no controle da verminose ovina: resultados parciais. PUBVET, Londrina, V. 5, N. 8, Ed. 155, Art. 1046, 2011.

\section{Introdução}

O rebanho ovino do Brasil representa apenas 1,7 do efetivo mundial. Considerando a dimensão territorial brasileira, bem como as condições edafoclimáticas favoráveis ao desenvolvimento da produção, nosso rebanho é numericamente inexpressivo. O sucesso de uma produção animal, em geral, depende das instalações, da interação genótipo-ambiente, do manejo, da alimentação e principalmente da saúde animal.

O parasitismo por nematóides gastrintestinais é considerado um obstáculo importante na criação de pequenos ruminantes, e as perdas causadas por esses parasitas estão relacionadas à baixa produtividade, diminuição do ganho de peso dos animais, diminuição no potencial materno, altos custos com tratamento e profilaxia e também morte de animais.

Dos parasitas de ovinos, a espécie Haemonchus contortus, destaca-se por encontrar-se em primeiro lugar na ordem de prevalência e de patogenicidade não só no Estado de São Paulo, mas em todo o território nacional. Em segundo lugar aparece a espécie Trichostrongylus colubriformis, mas na maioria das vezes as infecções são mistas sendo comum o parasitismo por espécies de Cooperia spp, Oesophagostomum spp e Strongyloides papillosus (AMARANTE, 2004).

O controle da verminose geralmente é realizado, através do uso de quimioterápicos, pertencentes a diversos grupos químicos, na maioria das vezes, administrados sem levar em consideração os fatores epidemiológicos da região, os quais interferem diretamente na população parasitária ambiental e, conseqüentemente, na reinfecção do rebanho.

A maioria dos produtores não adota esquema de vermifugação estratégico, nem realiza anualmente, de forma racional, a alternância dos grupos químicos utilizados, com isso, os endoparasitos rapidamente desenvolvem resistência às drogas disponíveis no mercado (RODRIGUES, 2005). 
ANDRADE, G.M., MOURA, M.S. e BARBOSA, F.C. Eficácia do produto homeopático Verm $100 \AA$ no controle da verminose ovina: resultados parciais. PUBVET, Londrina, V. 5, N. 8, Ed. 155, Art. 1046, 2011.

Os anti-helmínticos, em geral, não têm atividade prolongada, sendo comum a presença de parasitas um mês após a vermifugação, fato esse que pode ser devido a uma possível resistência dos parasitas às drogas utilizadas ou a grande infestação do meio ambiente por larvas. Além disso, cresce a demanda do consumidor por carne com qualidades organolépticas melhores, carne isenta, ou com o mínimo de resíduos de produtos químicos (ARO et al., 2006).

Métodos alternativos, que sejam de baixo custo e menos prejudiciais à saúde, animal e humana, e ao desequilíbrio ambiental estão sendo pesquisados e cada vez mais utilizados, como a seleção de animais resistentes, uso de fitoterápicos, fungos no controle ambiental e utilização de medicamentos homeopáticos.

A homeopatia, uma das alternativas para o controle de parasitas, faz o organismo trabalhar e criar anticorpos para se curar, atuando diretamente nas causas dos sintomas. Os medicamentos homeopáticos são feitos de plantas, animais e minerais (OLIVEIRA, 2005).

O princípio da homeopatia não é tratar as doenças, mas os animais, e os conceitos de energia vital, ação medicamentosa e reação do organismo, individualização terapêutica de rebanho, são essenciais.

O tratamento dos animais com o medicamento homeopático é feito em rebanho e tem vantagens como: equilíbrio animal (reduz o estresse de tratamentos convencionais melhorando o bem-estar animal), facilidade de administração, ausência de efeitos colaterais, inexistência de resíduos, ausência de contaminação do meio ambiente e baixo custo.

Segundo Furtado (2006), a demanda cada vez maior por produtos agropecuários livres de resíduos de produtos químicos, os chamados orgânicos, a intensa ocorrência de cepas de parasitas resistentes aos produtos químicos convencionais e a necessidade de se oportunizar melhores resultados para o pequeno pecuarista são fatores que favorecem a utilização de métodos alternativos no controle de parasitas gastrintestinais. 
ANDRADE, G.M., MOURA, M.S. e BARBOSA, F.C. Eficácia do produto homeopático Verm $100 \AA$ no controle da verminose ovina: resultados parciais. PUBVET, Londrina, V. 5, N. 8, Ed. 155, Art. 1046, 2011.

O presente estudo tem como objetivo avaliar a eficácia do produto homeopático VERM $100^{\circledR}$ (laboratório Real H) em ovinos, infectados naturalmente, mantidos a pasto, em uma propriedade no município de Prata, Minas Gerais.

\section{Material e métodos}

O estudo está sendo realizado em uma propriedade localizada no município de Prata, estado de Minas Gerais, com início em fevereiro de 2010.

O plantel é constituído de 50 ovinos mestiços Santa - Inês com idade variando de 6 meses a 36 meses, criados a pasto, em sistema semi-extensivo. Antes de começar a administração do medicamento foi realizado o exame de contagem do número de ovos por grama de fezes (OPG) em todos os animais e, posteriormente, divididos em dois grupos de vinte e cinco animais, de acordo com o OPG.

O grupo tratado, Grupo 1, recebeu $10 \mathrm{~g} /$ animal / dia do produto homeopático VERM $100 \AA$ (de acordo com as recomendações do fabricante, Laboratório Real $\mathrm{H}$ ), misturado ao sal mineral.

Já o grupo controle (Grupo 2) recebeu sal mineral, nas mesmas condições, entretanto, sem a adição de medicamento.

Mensalmente, após inicio do tratamento, foi feita a coleta de amostras de fezes de cada animal, diretamente do reto, para a realização da contagem de ovos por grama de fezes (OPG), segundo a técnica modificada de Gordon e Whitlock (1939).

Os resultados foram submetidos à Análise de Variância.

\section{Resultados e Discussão}

Os exames realizados no $D_{0}$ revelaram que os animais apresentavam OPG médio de $611,5 \pm 1177,2$ e 596, $2 \pm 1130,5$ para o grupo tratado e controle, respectivamente; grau de parasitismo considerado moderado por Ueno e Gonçalves (1998), tabela 1. 
ANDRADE, G.M., MOURA, M.S. e BARBOSA, F.C. Eficácia do produto homeopático Verm $100 \AA$ no controle da verminose ovina: resultados parciais. PUBVET, Londrina, V. 5, N. 8, Ed. 155, Art. 1046, 2011.

Tabela 1 - Média das contagens de ovos de estrongilídeos por grama de fezes (OPG) em ovinos segundo os tratamentos.

\begin{tabular}{llccc}
\hline & \multicolumn{3}{l}{ Média dos OPG e Desvio Padrão / Dias de tratamento } \\
\cline { 2 - 5 } Tratamento & Zero & +28 & +56 & +84 \\
\cline { 2 - 5 } Grupo I & $611,5 \pm 1177,2$ & $1653,8 \pm 2502,0$ & $811,5 \pm 1275,7$ & $546,2 \pm 874,6$ \\
Grupo II & $596,2 \pm 1130,5$ & $1219,2 \pm 3385,6$ & $415,4 \pm 1010,6$ & $176,9 \pm 250,3$ \\
\hline
\end{tabular}

$\mathrm{Na}$ avaliação do dia $\mathrm{D}_{28}$, observou-se o aumento no número médio de ovos por grama de fezes, tanto no grupo tratado quanto controle, com médias de $1653,8 \pm 2502,0$ e $1219,2 \pm 3385,6$, respectivamente. Os valores encontrados evidenciam a necessidade do tratamento anti-parasitário, e a não eficácia do tratamento.

Nos dias $D_{56}$ e $D_{84}$ houve a redução progressiva no número médio do OPG, para o grupo tratado e controle.

A redução no OPG, principalmente no grupo controle, possivelmente, se deve as mudanças de manejo como separação dos grupos, com melhores condições nutricionais dos animais e maior resistência as infecções parasitárias.

A análise estatística demonstrou que os grupos tiveram comportamentos semelhantes durante o período avaliado.

O gráfico 1 , sugere que, independente do animal estar no grupo tratado ou controle, em média a diminuição do número de ovos foi a mesma. Sendo que o grupo tratado teve maior número médio de OPG no início, e também ao final $\left(D_{84}\right)$.

No $D_{84}$ o grupo tratado apresentou OPG semelhante ao do dia $D_{0}$, e superior ao grupo controle que teve redução para 176,9 $\pm 250,3$ em média, infecção considerada leve. 
ANDRADE, G.M., MOURA, M.S. e BARBOSA, F.C. Eficácia do produto homeopático Verm $100 \AA$ no controle da verminose ovina: resultados parciais. PUBVET, Londrina, V. 5, N. 8, Ed. 155, Art. 1046, 2011.

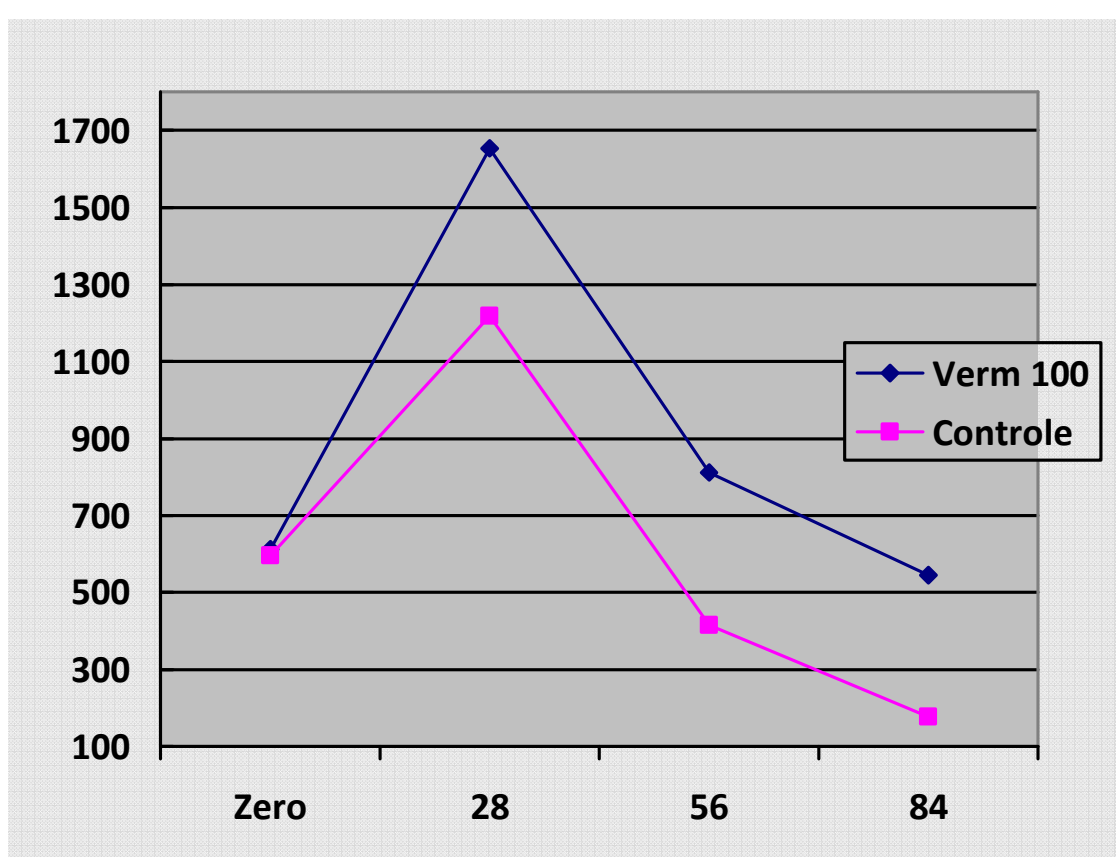

Gráfico 1 - Médias da contagem de ovos de estrongilídeos por grama de fezes (OPG) em ovinos segundo o tempo de exposição ao medicamento homeopático.

Poucos estudos avaliaram o uso de medicamentos homeopáticos no controle das parasitoses em ovinos (ROCHA et al., 2006).

Estes resultados coincidem com os de Cabaret (1996) e Rocha et al. (2006), que utilizaram Cina e o Fator Verme ${ }^{\circledR}$, respectivamente, e não encontraram nenhum efeito anti-helmíntico. Mas são contrários aos encontrados de Zeola et al. (2007), no qual a utilização do medicamento homeopático (Fator Vermes $\left({ }^{\circledR}\right)$ mostrou-se eficaz na redução do número de ovos por grama de fezes em de ovelhas no período de gestação.

Os animais do grupo tratado, não apresentaram redução na contagem de ovos por grama de fezes (OPG), mas também não demonstraram sinais clínicos de parasitose, pois segundo Cabaret et al. (2002), medicamentos homeopáticos parecem não ter ação antiparasitária, mas auxiliam o equilíbrio entre o parasito e o hospedeiro, reduzindo os efeitos da infecção. 
ANDRADE, G.M., MOURA, M.S. e BARBOSA, F.C. Eficácia do produto homeopático Verm $100 \circledast$ no controle da verminose ovina: resultados parciais. PUBVET, Londrina, V. 5, N. 8, Ed. 155, Art. 1046, 2011.

Embora o medicamento homeopático, nas condições deste estudo e no período avaliado, não tenha demonstrado eficaz, os animais do grupo tratado mostraram-se visualmente mais saudáveis e com maior ganho de peso, quando comparado ao grupo controle, embora não tenha realizado avaliação do escore corporal.

\section{Conclusão}

De acordo com a metodologia utilizada e o período de avaliação, os resultados obtidos são insuficientes para poder concluir se o medicamento VERM $100 \circledR$ é eficaz no controle da verminose ovina.

Sugere-se que o período de avaliação deva ser maior, pois os efeitos no controle das parasitoses com produtos homeopáticos, possivelmente, sejam observados a longo prazo.

\section{Referências}

AMARANTE, A. F. T. Controle da Verminose Gastrointestinal no Sistema de Produção de São Paulo, I Congresso Brasileiro de Especialidades em Medicina Veterinária. Paraná, 2004.

ARENALES, M.C., 2002. Homeopathy in cut cattle. In: Global Virtual Conference on Organic Production of Bovines of Cut, University of Constestado, UnC, Concordia Unit, 2 set -15 out 2002, 11pp.

ARO, D. T.; POLIZER, K. A.; BELUTE, D. S.; ALMEIDA, C. R. de; AMARAL, L. C. do; NEVES, M. F.; RODRIGUES, R. Verminose Ovina. Revista Científica Eletrônica de Medicina Veterinária. Garça, v. III, n.07, jun. 2006.

CABARET, J. The homeopathic Cina does not reduce the egg output of digestive-tract nematodes in lambs. Revuede Medecine Veterinaire, v.147, n.6, p.445-446, 1996.

CABARET, J.; BOUILHOL, M.; MAGE, C. Managing helminths of ruminants in organic farming. Veterinary Research v.33, n.5, p.625-640, 2002.

CHAGAS, A. C. S.; VIEIRA, L. S.; FREITAS,A. R.; ARAÚJO, M. F. A.; ARAÚJO-Filho, J. A.; ARAGUA, W. R.; NAVARRO, A. M. C. Anthelmintic efficacy of neem (Azadirachta indica A. Juss) and the homeopathic product Fator Vermes in Morada Nova sheep. Veterinary Parasitology, v. 151, p. $68-73,2008$.

CRUZ, J. F.; VIANA, A. E. S.; OLIVEIRA, D. F.; FERRAZ, R. C. N.; MAGALHÃES, M. P.; SANTOS, D. D.; CRUZ, R. S.; CRUZ, A. D.; ZACHARIAS, F. A homeopatia como ferramenta de controle de helmintos gastrintestinais em caprinos criados em sistema extensivo. A Hora Vet, Caçapava do Sul, v.154, p.37-40, 2006. 
ANDRADE, G.M., MOURA, M.S. e BARBOSA, F.C. Eficácia do produto homeopático Verm $100 \circledast$ no controle da verminose ovina: resultados parciais. PUBVET, Londrina, V. 5, N. 8, Ed. 155, Art. 1046, 2011.

FURTADO, S. K. Alternativas fitoterápicas para o controle da verminose ovina no estado do Paraná: Teste in vitro e in vivo. 2006.147f. Tese (Doutorado em Ciências Agrárias) - Departamento de Fitotecnia e Fitossanitarismo, Setor de Ciências Agrárias, Universidade Federal do Paraná, Curitiba, 2006.

GORDON, H.M.; WHITLOCK, H.V. A. New technique for counting nematodes eggs in sheep faeces. J. Coun. Sci. Ind. Res. Aust., v.12, p.50- 52, 1939

HAHNEMANN, S. Organon da arte de curar. 6. ed. São Paulo: Indústria Gráfica e Editora Ltda, 1996.

OLIVEIRA, S. M. Triagem de medicamentos homeopáticos que ativam macrófagos com detecção e quantificação de IFN-g, IL-4 e NO. 2008. 122f. Dissertação (Mestrado em Biologia Celular e Molecular) - Departamento de Biologia Celular do Setor de Ciências Biológicas, Universidade Federal do Paraná, Curitiba, 2005.

ROCHA, R.A.; PACHECO, R.D.L.; AMARANTE, A.F.T. Efficacy of homeopathic treatment against natural infection of sheep by gastrointestinal nematodes. Rev.Bras. Parasitolol. Vet, v.15, n.1, p. 23-27, Botucatu, 2006.

RODRIGUES, A. B. Sensibilidade dos Nematóides gastrintestinais de caprinos e ovinos a anti-helmínticos na mesoregião do Sertão Paraibano. 2005. 92f. Dissertação (Mestrado em Ciências Agrárias na área de Epidemiologia das Doenças Parasitárias) - Faculdade de Medicina Veterinária de Pequenos Ruminantes do Centro de Saúde e Tecnologia Rural. Universidade Federal de Campina Grande, Patos, 2005.

SOUZA, M. F. A. Homeopatia Veterinária. I Conferência Virtual Global sobre Produção Orgânica de Bovinos de Corte 02 de setembro à 15 de outubro de 2002, Brasil.

TEIXEIRA, M. M. O uso do Nim (Azadirachta indica A. Juss.) como alternativa fitoterápica no controle de encoparasitas de pequenos ruminantes. 2008. $29 \mathrm{f}$. Monografia - Faculdade de Medicina Veterinária, Universidade Federal de Uberlândia, Uberlândia, 2008.

UENO, H.; GONÇALVES, P. C. Manual para diagnóstico de helmintoses de ruminantes. $4^{\mathrm{a}}$ ed. Tokio: Japan international Cooperation Agency, 1998. 143 p.

VIEIRA, L. S.; CAVALCANTE, A. C. R.; PEREIRA, M. F.; DANTAS, L. B; XIMENES, L. J. F. Evaluation of anthelmintic efficacy of plants avaiable in Ceará State, North - East Brazil, for the control of goat gastrointestinal nematodes. Revue de Medecine Veterinaire, Toulouse, v. 150, n. 5, p. 447-452, 1999.

ZEOLA, N. M. B. L.; SOBRINHO, A. G. S.; LEÃO, A. G.; PEREZ, H. L.; SANTOS, E. S. dos. Homeopatia no controle de helmintos gastrintestinais de ovelhas em gestação. 44a Reunião Anual da Sociedade Brasileira de Zootecnia, Jaboticabal. 24 a 27 jul 2007. Disponível em: http://www.arenales.com.br/biblioteca/Reuniao_anual_soc_bras_zootecnia.pdf Acesso em: 10 mai. 2010. 